\title{
PROJECTIVE INVARIANTS OF PROJECTIVE STRUCTURES AND APPLICATIONS
}

\author{
By DAVID MUMFORD
}

The basic problem that I wish to discuss is this: if $V$ is a variety, or scheme, parametrizing the set, or functor, of all structures of some type in projective $n$-space $\mathbf{P}_{n}$, then the group $P G L(n)$ of automorphisms of $\mathbf{P}_{n}$ acts on $V$. Then under what conditions does there exist a quotient or orbit space $V / P G L(n)$, i.e. when can we construct enough "projective invariants" for these structures? For example, let $V$ parametrize the set of hypersurfaces of degree $m$, with certain types of singularities; or let $V$ parametrize the set of tri-canonical space curves of given genus, or even $n$-canonical surfaces with at most "negligible singularities" [1]; or let $V$ parametrize the set of 0 -cycles of degree $m$ in $\mathbf{P}_{n}$; or let $V$ parametrize the set of all morphisms of a fixed scheme $X$ into $\mathbf{P}_{n}$. Moreover, I wish to illustrate how such questions are one essential step in several basic existence and construction problems of algebraic geometry.

One approach to this problem is afforded by the invariant theory of the representations of reductive groups. Here you generalize the problem first: consider an arbitrary action of an algebraic group $G$ on a variety (or scheme) $V$ and seek an orbit space $V / G$. Then you specialize the problem by $(a)$ assuming $G$ is reductive, $(b)$ restricting attention to quasi-projective orbit spaces $V / G$. Of course, if, in particular, the characteristic is $0, P G L(n)$ is reductive. Now suppose you have a projective embedding $V \subset \mathbf{P}_{N}$. If $V$ is a normal variety, and if this embedding is defined by a complete linear system on $V$, it is possible to prove that the action of $G$ on $V$ extends to an action of $G$ on $\mathbf{P}_{N}$. In any case, if this occurs, I say that $G$ acts linearly on $V \subset \mathbf{P}_{N}$.

Now, if the action of $G$ is linear, its action is induced by a linear and unimodular representation of some finite covering $G^{*}$ of $G$ on the affine cone $\mathbf{A}^{N+1}$ over the ambient $\mathbf{P}_{N}$. Then I make the definition:

A point $x \in V$ is stable for the action of $G$ on $V$, relative to the embedding $V \subset \mathbf{P}_{N}$, if for one (and hence all) homogeneous points $x^{*} \in \mathbf{A}^{N+1}$ over $x$, (i) the stabilizer of $x^{*}$ is a finite subgroup of $G^{*}$, and (ii) the orbit of $x^{*}$ under $G^{*}$ is closed in $\mathbf{A}^{N+1}$.

Now assume that a reductive algebraic group $G$ acts linearly on $V \subset \mathbf{P}_{N}$. The fundamental theorem is this:

Theorem. The set of stable points forms an open set $U$ in $V$, and a quasiprojective orbit space $U / G$ exists.

In case $G$ is semi-simple and of characteristic 0 , I can say more. First, let us call the action $\alpha: G \times V \rightarrow V$ of $G$ on $V$ a proper action if the morphism $\alpha \times P r_{2}: G \times V \rightarrow V \times V$ is proper (see [2]). Then:

Theorem. (1) $A$ point $x \in V$ is stable if and only if there is a hypersurface section $H$ of $V$ which is invariant under $G$, and such that $x \notin H$ and the orbit of $x$ is closed in $\mathrm{V}-\mathrm{H}$.

(2) The action of $G$ on the set of stable points $U$ is proper. 
(3) If $G$ acts properly on $V$, and if a quasi-projective orbit space $V / G$ exists, then for some projective embedding $V \subset \mathbf{P}_{N}$, every point of $V$ is stable.

Finally, if $G$ is any reductive algebraic group in characteristic $O$, then I can analyze the manner in which stability breaks down in the following way:

Theorem. If $x \in V$ is not stable for the action of $G$, then there is a Borel subgroup $B \subset G$ and a 1-parameter subgroup $\mathbf{G}_{m} \subset B$ such that, if $\mathbf{G}_{m}$ is any conjugate of $\mathbf{G}_{m}$ in $B$, then $x$ is not stable for the action of $\mathbf{G}_{m}$ on $V$.

These results are not definitive: I conjecture that they all are valid for semi-simple groups of any characteristic. However, they suggest the kind of answer that should be found for the original question: to every type of structure in $\mathbf{P}_{n}$, there is a stability condition which is sufficient and, in general, necessary for the existence of enough projective invariants to classify these structures; and, moreover, this stability condition is always of the form: there is no flag in $\mathbf{P}_{n}$ which has "too high an order of contact" with the given structure.

The following case has been worked out exhaustively by myself and $J$. Tate in all characteristics, and even over the scheme of integers: let $V=$ $\left(\mathbf{P}_{n}\right)^{m}$, i.e. $V$ parametrizes ordered 0 -cycles of degree $m$ in $\mathbf{P}_{n}$. Then relative to the Segre embedding of $V$, a point $x=\left(x_{1}, x_{2}, \ldots ., x_{m}\right)$ is stable if and only if:

For all linear subspaces $\mathrm{L} \subset \mathbf{P}_{n}$, then:

$$
\frac{\left(\text { number of points } x_{i} \text { in subspace } L\right)}{\left(\text { total number of points } x_{i}\right)}<\frac{\operatorname{dim}(L)+1}{n+1} .
$$

Then in all characteristics, or even over the scheme of integers, the set of stable 0-cycles forms an open set $U \subset\left(\mathbf{P}_{n}\right)^{m}$, and a quasi-projective orbit space $U / P G L(n)$ exists. In fact, $U$ is a principle fibre bundle over $U / P G L(n)$. Our techniques are entirely elementary (see [3] and [6]).

I would like to illustrate how this simple result can be used to prove the existence of (i) a moduli scheme for polarized abelian varieties, and (ii) (according to a suggestion of Grothendieck) the Picard scheme of any variety. $\left(^{1}\right)$ First, let me fix some notations: if $U \subset\left(\mathbf{P}_{n}\right)^{m}$ is as above, let $Q_{n, m}=U / P G L(n)$. Second, since $U$ is a principal fibre bundle over $Q_{n, m}$ with group $P G L(n)$, if $P G L(n)$ acts on any scheme $X$, we can form an associated fibre bundle with fibre $X$; in particular, if $X=\mathbf{P}_{n}$, denote the associated fibre bundle by $P_{n, m}$. Let $\pi: P_{n, m} \rightarrow Q_{n, m}$ be the bundle morphism. It is easy to see that the bundle $P_{n, m} / Q_{n, m}$ has $m$ distinguished sections $s_{i}: Q_{n, m} \rightarrow$ $P_{n, m}$ associated to the maps which take the $m$-tuple $\left(x_{1}, x_{2}, \ldots, x_{m}\right)$ to its ith factor $x_{i}$. Intuitively, regard $P_{n, m}$ plus the collection of sections $\left(s_{i}\right)$ as the universal family of projective $n$-spaces with given stable 0 -cycle of degree $m$.

To simplify the treatment of the moduli problem, let us consider only the question of finding a quasi-projective variety $M_{g, d}$ whose points parametrize "naturally" the set of all abelian varieties $A$ of dimension $g$, plus a very ample $\left({ }^{2}\right)$ divisor class $D$ such that $\left(D^{g}\right)=d \cdot g$ ! For the question of

(1) In fact, it can be used to prove much stronger results on the existence of relative Picard schemes: see [4].

$\left({ }^{2}\right)$ in the sense of Grothendieck, i.e. induced via a projective embedding. 
making explicit the sense of the word "naturally", and the question of replacing $D$ by its numerical equivalence class, see [6]. Now pick any $n$ such that the characteristic does not divide $n$, and $n>d \cdot \sqrt{g !}$. Let $\nu=n^{2 \sigma}$. Then consider the following set:

Subvarieties $A \subset P_{d-1, v}$ such that

(a) $\pi(A)$ is a single point $q \in Q_{d-1, v}$,

(b) the degree of $A$ in $\pi^{-1}(q)$ equals $d \cdot g$ !, and the dimension of $A$ is $g$,

(c) A admits a structure of abelian variety such that $\left\{s_{i}(q)\right\}$, for $i=$ $1,2, \ldots, v$ is the set of points of order $n$.

Then, on the one hand, this set is parametrized naturally by a locally closed subset of a suitable Chow variety. But, on the other hand, it is isomorphic to the set of abelian varieties $A$ of dimension $g$, plus a very ample divisor class $D$ such that $\left(D^{g}\right)=d \cdot g !$, plus an ordering of the set of points of order $n$. [Namely, one can prove that the inequality $n>d \cdot \sqrt{g}$ ! insures that the set of points of order $n$ form a stable 0 -cycle in $\mathbf{P}_{d-1}$ via the embedding defined by the complete linear system $|D|$. Let $q$ be the equivalence class of this 0 -cycle mod $P G L(d-1)$; then there is a unique identification of $\pi^{-1}(q)$ with the $\mathbf{P}_{d-1}$ ambient to $A$, under which $s_{i}(q)$ corresponds to the $i$ th point of order $n$. Then $A$ is embedded in $\pi^{-1}(q) \subset P_{d-1, v}$ via this identification.] Therefore, the set of all $A$, plus $D$ alone, is parametrized by the quotient of this set by the group of permutations of $v$ letters. As such a quotient of a quasi-projective variety is well-known to exist, this solves the problem.

To simplify the treatment of the Picard scheme of a projective variety $V$, let us consider only the construction of the reduced and connected Picard scheme. The question then may be loosely described as that of finding a variety $P$ whose points parametrize "naturally" the set of all Cartier divisor classes $D$ on $V$ which are algebraically equivalent to 0 . But this is equivalent to doing the same for the set of all Cartier divisor classes $D$ on $V$ which are algebraically equivalent to some fixed very ample divisor $D_{0}$. But if $D_{0}$ is chosen sufficiently ample, and if we associate to each divisor class $D$ the morphism $\phi: V \rightarrow \mathbf{P}_{n}$ defined by the complete linear system $|D|$, then this set will be isomorphic to the set of orbits under the group $P G L(n)$ in the set of morphisms $\phi: V \rightarrow \mathbf{P}_{n}$ which are algebraically equivalent to a fixed $\phi_{0}: V \rightarrow \mathbf{P}_{n}$. This is a problem of the type originally posed.

It can be reduced to the 0 -cycle problem already solved as follows: pick a large number of sufficiently generic points $x_{1}, x_{2}, \ldots x_{N}$ in $V$, i.e. such that:

For all $\phi: V \rightarrow \mathbf{P}_{n}$ algebraically equivalent to $\phi_{0}$, the set of points $\phi x_{1}$, $\phi x_{2}, \ldots, \phi x_{N}$ is a stable 0 -cycle in $\mathbf{P}_{n}$.

Then consider the following set:

Morphisms $\phi: V \rightarrow P_{n, N}$ such that

(a) $\pi \circ \phi$ maps $V$ to a single point $q \in Q_{n_{\mathrm{v}} N}$,

(b) $s_{i}(q)=\phi\left(x_{i}\right)$,

(c) $\phi$, as a morphism from $V$ to $\pi^{-1}(q) \cong \mathbf{P}_{n}$ is algebraically equivalent to $\phi_{0}$.

Then on the one hand this set is parametrized by a locally closed subset of a suitable Chow variety (i.e. via the graph of $\phi$ ), and on the other hand it is isomorphic to the set of orbits described above. 
Here is another example of a stability condition and the resulting quotient theorem. Assume the characteristic is 0 , and consider, instead of sequences of points in $\mathbf{P}_{n}$, sequences of linear subspaces of any dimension. Thus, if Grass $_{k, n}$ stands for the Grassmannian of $k$-dimensional linear subspaces of $\mathbf{P}_{n}$, I ask for orbit spaces of the type $\left(G_{r a s s}, n\right)^{m} / P G L(n)$. Then, in fact, relative to the usual Plücker embedding of the Grassmannian, and to the Segre embedding of this product, it turns out that a point $x=\left(L_{1}, L_{2}, \ldots, L_{m}\right)$ of $\left(\text { Grass }_{k, n}\right)^{m}$ is stable if and only if:

For all linear subspaces $L \subset P_{n}$, then:

$$
\frac{\sum_{i}\left(\operatorname{dim}\left(L \cap L_{i}\right)+1\right)}{\sum_{i}\left(\operatorname{dim} L_{i}+1\right)}<\frac{(\operatorname{dim} L+1)}{(n+1)} .
$$

Then, by the fundamental theorem, the set of stable points forms an open set $U$, and an orbit space $U / P G L(n)$ exists.

This result can be applied to the problem of classifying vector bundles over a variety in exactly the same way as the result on 0-cycles was applied to the problem of classifying line bundles, i.e. Cartier divisor classes. Of course, it is well-known that the set of vector bundles even over an algebraic curve is not a separated space; in fact, it is not even locally separated, because of the "jump" phenomenon noted by Kodaira and Spencer [5]. However, again, a basic stability condition avoids all these difficulties. For simplicity, let us consider only vector bundles over a fixed-curve $C$.

Definition. A vector bundle $E$ is stable if for all sub-bundles $F$,

$$
\operatorname{Deg} c_{1}(F)<\operatorname{Deg} c_{1}(\mathrm{E}) \cdot \frac{\operatorname{rank} F}{\operatorname{rank} E},
$$

where $c_{1}$ denotes the first chern class.

In other words, a vector bundle is stable if all its subbundles are "less ample" than itself. To illustrate the stability condition, let me mention its simplest properties:

(i) If $L$ is a line bundle, then $E$ is stable if and only if $E \otimes L$ is stable; moreover, $E$ is stable if and only if $\breve{E}$ is stable.

(ii) If $E_{1}$ and $E_{2}$ are two vector bundles, $E_{1} \oplus E_{2}$ is never stable.

(iii) A line bundle is always stable.

(iv) If a vector bundle $E$ of rank 2 is not stable, then either $E$ is isomorphic to $L_{1} \oplus L_{2}$, or there is a unique sub-bundle $L$ for which $\geqslant$ holds in the definition and $\boldsymbol{E}$ can be canonically described as an extension.

Then I can prove the following theorem:

Theorem. The set of all stable vector bundles of rank $\mathrm{r}$ over a fixed curve $\mathrm{C}$ in characteristic 0 is "naturally" isomorphic to the set of points of a nonsingular quasi-projective variety $V_{r}(\mathrm{C})$.

A more complicated example of a stability condition is given by the action of $P G L(2)$ on the variety of plane curves of degree $n$. There seems to be no simple general rule describing when a plane curve is stable; however, I can prove that if $n \geqslant 3$, then at least every non-singular curve is stable. For low values of $n$, the precise answer is given by: 


\section{Stable curves}

None.

Non-singular.

No triple points and no tacnodes.

No triple points with 3 coincident tangents, or with 2 tangents forming a tacnode.

To conclude, I want to pose a question that seems to me to be the most interesting problem in extending the results discussed above. Moreover, I think this problem is the central one on the road to solving the general problem of algebraic moduli of polarized non-singular varieties. The question is: when is the Chow point of a non-singular subvariety $\mathrm{V} \subset \mathbf{P}_{n}$ stable (in the usual projective embedding of the Chow variety)? Perhaps more reasonable is the stabilized form of this problem: Given a subvariety $V \subset \mathbf{P}_{n}$, when is there an $n_{0}$ such that if $n \geqslant n_{0}$, the Chow point of $V$ in the $n$-ple embedding $V \subset \mathbf{P}_{N}$ is stable? I have no conjecture to make.

\section{REFERENCES}

[1]. Artin, M., Some numerical criteria for the contractability of curves on an algebraic surface. Amer. J. Math., 84 (1962), 485.

[2]. Palats, R., On the existence of slices for actions of non-compact Lie groups. Ann. Math., 73 (1961), 295.

[3]. MuMFord, D., An elementary theorem in geometric invariant theory. Bull. Amer. Math. Soc., 67 (1961), 483.

[4]. GrothendeCK, A., Séminaire Bourbaki, exp. 232 and 236, 1962.

[5]. Kodatra, K. \& Spencer, D. C., On deformations of complex analytic structures. Ann. Math., 67 (1958), 328.

Most of the material discussed above will be published in:

[6]. MUMFord, D., Geometric invariant theory. (Forthcoming.) 